\title{
FREEZING OF ORTHOPAEDIC SPECIMENS BEFORE MECHANICAL TESTING
}

\author{
Z. A. RALIS \\ From the University of Wales College of Medicine, Cardiff
}

\begin{abstract}
The effect of storage at sub-zero temperatures and subsequent thawing was investigated in dissected muscles, tendons, limbs and spines. Freezing caused a noticeable shortening of muscles which when thawed could easily be elongated; the same effects, though less pronounced, were observed with tendons. During freezing, myotomy or tenotomy led to the development of a striking deformity owing to unopposed shortening of the opposing muscles. After thawing, all frozen specimens containing muscles and joints showed an increased range of passive movements, easily demonstrable by mild tensile forces.
\end{abstract}

In biomechanical studies of locomotor system tissues post-mortem specimens often have to be preserved for some time before testing. Freezing is often chosen as the method of preservation; the specimens can be kept indefinitely and thawed when needed. Although there are reports that freezing temperatures do not affect the mechanical properties of cortical bone (Sedlin 1965) or cartilage (Lee and Pelker 1985) the destructive effect of freezing on muscle tissue is known, for example, in cryobiology and the meat industry. Rapid freezing to very low temperatures such as $-150^{\circ} \mathrm{C}$ results in the formation of small intercellular crystals, whereas freezing to temperatures between $-15^{\circ} \mathrm{C}$ and $-60^{\circ} \mathrm{C}$ (the range in which most commercial freezers operate) leads to the formation of large ice cavities which destroy the cytoplasmic membranes and disrupt the architecture of both the sarcomere and myofibrils (Menz 1971). The author has recorded that individual skeletal muscles which shortened during freezing elongated after thawing and that there was a similar though less pronounced effect with tendons (unpublished). Other locomotor system tissues that contain fluid, such as joint capsules, synovia and ligaments, are also likely to be affected by freezing. The effect of freezing was studied on the mechanical properties of orthopaedic specimens which contained all the main tissues (bone, cartilage, muscles, ligaments and joint tissues).

\section{MATERIAL AND METHODS}

Twelve adult New Zealand white rabbits and four adult white Wistar rats were dissected post mortem after rigor mortis had passed. Nine lower limbs, four upper limbs,

Z. A. Ralis, MD, Senior Lecturer and Honorary Consultant Department of Traumatic and Orthopaedic Surgery, University of Wales College of Medicine, Cardiff Royal Infirmary, Cardiff CF2 ISZ, Wales.

(C) 1989 British Editorial Society of Bone and Joint Surgery $0301-620 X / 89 / 1015 \$ 2.00$

J Bone Joint Surg [Br] 1989;71-B:55-7. seven individual muscles and tendons and five dissected spines were removed. In addition, four human postmortem muscle specimens were examined. After preparation and initial measurements the specimens were stored for four days in a conventional deep freeze operating at a constant $-24^{\circ} \mathrm{C}$, where they were suspended freely from a bar to facilitate any changes in their dimension and shape. After removal from the deep freeze the specimens were measured frozen; they were then immersed in saline and left to thaw at room temperature. Finally, they were blotted dry and measured again.

The specimens were tested in four ways:

a) Long-fibred human muscles and tendons, including rectus abdominis, psoas, the long erector of the spine, and the tendons of the long toe extensor and flexor were dissected. Before they were frozen a black silk stitch was inserted at the proximal and distal ends of each muscle or tendon. Each stitch also formed a fixed loop; the proximal loop was pinned firmly to the cork board on which the muscle specimen had been placed and the distal loop was connected to an ordinary rubber band which when extended by a fifth of its length exerted a uniform $300 \mathrm{~g}$ tension. The band was then pinned through another silk loop to the cork board distally. The muscle specimen was thus stretched under a mild, controlled and equal tension. The length (the distance between the proximal and distal stitches) of the specimen was measured after one minute, when the specimen had settled. After freezing and thawing the specimens were again stretched and measured as before. The tendons were measured in the same way except that a tensile force of $1,000 \mathrm{~g}$ for two minutes was used.

b) In each of six limbs dissected from rabbits and rats the distal tendon of one of the following muscles was cut: long flexor of the paw, quadriceps femoris, tibialis anterior, long toe extensor, gastrocnemius and long toe flexor. After tenotomy the position of each part of the suspended limb was noted before it was hung in the deep freeze.

c) The lower extremities of five rabbits were dissected 
together with the lower part of the trunk. Into the trunk, and into the lower end of the tibia, two strong pins were inserted as proximal and distal anchorages for a rubber band (Fig. 1). The rubber bands stretched between the two pins exerted a tension of 650 to $850 \mathrm{~g}$ and forced the limb into maximum flexion. After two minutes for the specimen to settle the distances between the two pins and the angles of the flexed hip and knee joints were measured. The rubber bands were then removed, and the specimens frozen; when thawed the procedure was repeated with the same rubber bands and the measurements were taken again.

d) In five rabbits all or most of the spine was dissected and strong pins were fixed into the proximal and distal ends as attachments for a rubber band. In this way, under a tension of $850 \mathrm{~g}$, the specimen was bent either into flexion (three spines) or into extension (two spines) (Fig. 2). The distance between the two pins was measured and after freezing and thawing the procedure and measurements were repeated.

\section{RESULTS}

Table I shows the main results of freezing and thawing on the specimens tested. Individual dissected muscles when frozen shortened by over $10 \%$, but after thawing they lengthened by about $8 \%$ of their original length. The dissected tendons showed changes similar to those of muscle - particularly the increase in length after thawing. Tenotomy or myotomy in all specimens led to a pronounced deformity produced by the intact, frozen and shortened, opposing muscles (Fig. 3).

The deformities were always pronounced, with the exception of two specimens in which the calcaneal tendon was cut. Unopposed contractions of the antagonists of the muscles that were cut caused deformity in frozen specimens. During freezing the foot started to move upwards but stopped when all the muscles froze together. Where the hips and knees were forced by the rubber band into flexion, even more acute bending was achieved after freezing and thawing. Two such limbs were afterwards frozen and thawed for a second time: the maximum flexion increased by another 2 to $3^{\circ}$. The dissected spines curved by the rubber bands also showed a greater readiness to bend when they had been frozen and thawed.

\section{DISCUSSION}

The influence of sub-zero temperatures on biological tissues is studied in cryobiology, a specialty mainly concerned with the preservation of viability of cells in tissues during storage. Cell and tissue destruction have been prevented by the use of programmed freezing, controlled freezing rates and cryoprotective agents. For example, in articular cartilage grafts more than $50 \%$ cellular survival can be achieved (Schachar and McGann

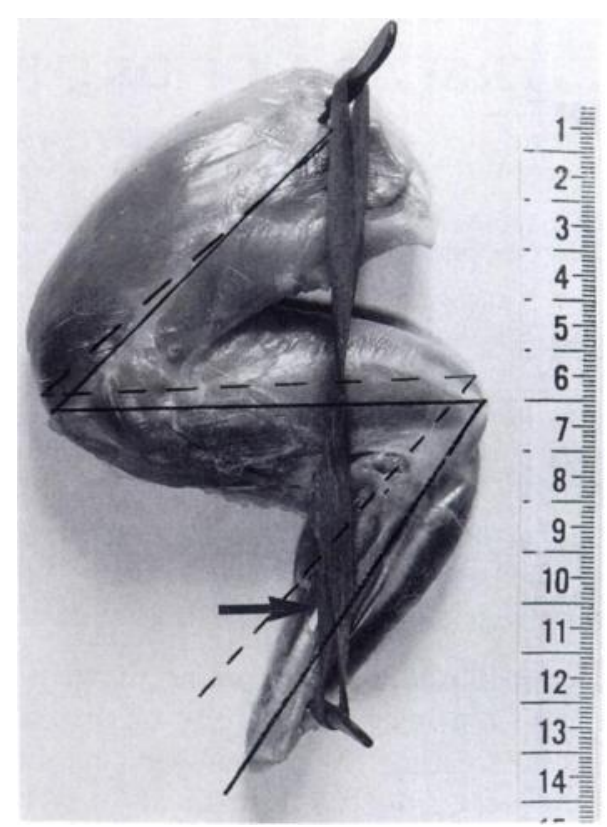

Fig. 1

The lower part of a rabbit trunk and hind limb showing the hip and knee joints forced into passive flexion by a rubber band (tensile force $650 \mathrm{~g}$ ). The unbroken line indicates the position of the joints before freezing, the broken line and arrow shows the new position of the distal pin and more pronounced joint flexion after thawing.

1986). In cryosurgery, however, the aim is effective destruction of pathological lesions with preservation of viability of the surrounding tissues.

There are few reports about the changes in mechanical properties of tissues that have been frozen. Lee and Pelker (1985) studied the effects of freezing and thawing on the failure of the capital femoral growth plate of the rabbit under shear loads and stresses and did not find any significant differences. However, they conceded that the perichondrial ring had been removed from their test specimens, which may have affected the results. Yet in studies on the cryopreservation of cartilage it is accepted that during freezing not only the vitality of the cells is threatened but other damage also takes place, such as the formation of extracellular ice crystals, intracellular nucleation of ice, matrix disruption and the breakage of intercellular bridges (Schachar and McGann 1986).

The changes seen in muscles after freezing and thawing were expected: a visible and measurable shortening during freezing and elongation after thawing. However, much the same but less expected changes were also observed in tendons. The acquired flaccidity of muscles after freezing and thawing is in accord with what is known about the destructive effect of the $-20^{\circ}$ temperature at which specimens were tested and are usually stored. Another factor should be considered: the contraction of intact muscles during the freezing process 
Table I. Effect of freezing $-24 \mathrm{C}$ and thawing on the specimens

\section{Method* Specimen tested \\ (a) Dissected muscle (human and rabbit) Dissected tendon (rabbit) \\ (b) Muscle tenotomy in limb in situ (rabbits and rats) \\ Frozen \\ Mean shortening $11.4^{\circ}$ 。 Mean shortening $8.15^{\circ}$ 。 \\ Pronounced deformity of the elbow (knee) or paw (foot) produced by opposing muscles

\author{
After thawing \\ Mean lengthening $7.97^{\circ}$ 。 \\ Both cut and intact muscles \\ and $\operatorname{limb}$ are foppy
} Mean lengthening $6.75^{\circ} \%$}

(c) Intact limb with forced flexion of the hip and knee (rabbits)

(d) Forced bending of the dissected spine (rabbits)

* See text for description of methods (a) to (d).
Distance between proximal and distal rubber band attachments shortened by $19.33^{\circ}$, causing increase of the hip and knee flexion by $5^{\circ}$ and $12^{\circ}$ o respectively

Distance between proximal and distal rubber band attachments shortened by an average $15^{\circ}$, causing more acute bending

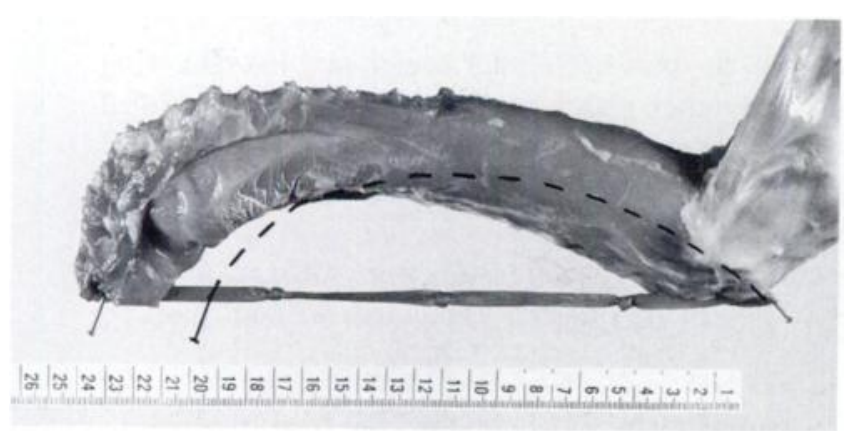

Fig. 2

A rabbit spine forcibly bent by a rubber band which exerted a tension of $850 \mathrm{~g}$ before freezing. The broken line indicates the increased range of bending after freezing and thawing of the specimen.

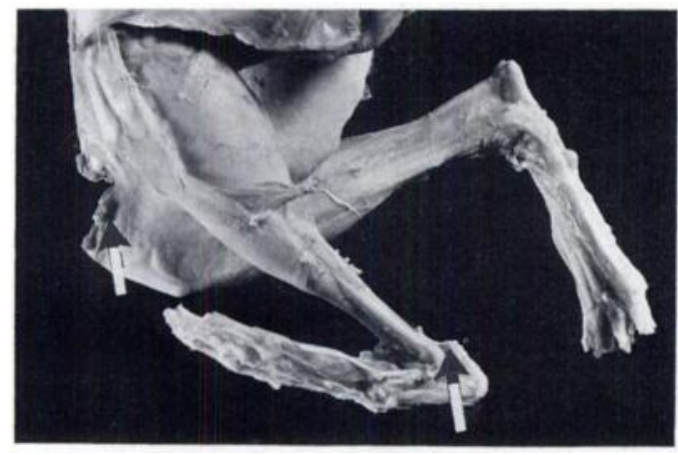

Fig. 3

Post-mortem myotomy was performed of the quadriceps femoris on the right hind limb of a rat and tenotomy of the calcaneal tendon on the left (arrows). During freezing there was pronounced knee flexion on the right side and knee extension with foot dorsiflexion on the left.

produced deformation of the specimen whenever the opposing muscles were sectioned or removed during dissection.

The increased range of passive flexion in hip and knee joints after thawing indicates a greater than usual laxity in the ligaments and joint structures which normally limit the range of passive movements. This increased laxity was especially obvious in the dissected spines from which most of the muscles had been removed, so that the increased bending reflected the influence of freezing on the joint capsules.

Conclusion If biomechanical tests on post-mortem specimens are to reflect conditions in life, specimens should be removed from the body as soon as rigor mortis has passed and tested immediately in the fresh state; even if these conditions are fulfilled, the ever present and ever changing tensile and compressive loads of living muscles, that normally act on the osseo-articular components, are lost. Once the specimens have been frozen and thawed two artefactual distortions occur. First, during freezing the contracted opposing muscles of those removed or cut during dissection will cause some flexion deformity in their direction. Secondly, after thawing and mechanical loading of the specimen the flaccid and elongated muscles and stretchable ligaments will cause greater deformability in all joints of the specimen.

This project was partly supported by the National Fund for Research into Crippling Diseases (Action Research), grant 1983 - 1985 and by ASBAH (London), grant 1983 - 1985. The author thanks Mrs Heather M. Ralis for editing and preparing the script.

No benefits in any form have been received or will be received from a commercial party related directly or indirectly to the subject of this article.

\section{REFERENCES}

Lee KE, Pelker RR. Effect of freezing on histologic and biomechanical failure patterns in the rabbit capital femoral growth plate. J Orthop Res $1985 ; 3: 514-5$

Menz LJ. Structural changes and impairment of function associated with freezing and thawing in muscle, nerve and leucocytes. Crybiology $1971 ; 8: 1-13$.

Schachar NS, McGann LR. Investigations of low-temperature storage on articular cartilage for transplantation. Clin Orthop $1986 ; 208: 146-50$.

Sedlin E. A rheologic model for cortical bone. Acta Orthop Scand (Suppl) 1965:36:83. 